Concept Paper

\title{
Understanding the Functional Roles of Multi-Modal Processing and Gc Activation In Older People's Performance in Caregiving Training
}

\author{
Robert Zheng *
}

Department of Educational Psychology, University of Utah, USA; E-Mail: robert.zheng@utah.edu

* Correspondence: Robert Zheng; E-Mail: robert.zheng@utah.edu

Academic Editor: James S. Powers

Special Issue: Models of Caregiver Support

\section{OBM Geriatrics}

2018 , volume 2 , issue 4

doi:10.21926/obm.geriatr.1804026
Received: October 05, 2018

Accepted: December 22, 2018

Published: December 26, 2018

\begin{abstract}
The current article addresses the working memory constraints experienced by older people in caregiving training. Two different approaches aiming to free the older people's working memory space were discussed. They include multi-modal visual processing and Gc activation. The emphasis was on the theoretical underpinnings of both approaches with empirical evidence from the research and literature to demonstrate their effectiveness in older people's caregiving training. Further discussion was made with respect to the theoretical and practical significance of the approaches. Suggestions were included with directions for future research.
\end{abstract}

\section{Keywords}

Caregiving training; cognitive prompts; g intelligence; multi-modal processing; older adult learning; schema activation

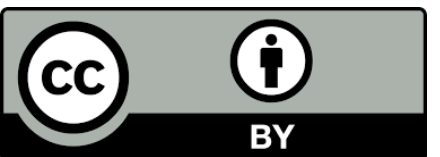

(C) 2018 by the author. This is an open access article distributed under the conditions of the Creative Commons by Attribution License, which permits unrestricted use, distribution, and reproduction in any medium or format, provided the original work is correctly cited. 
There are significant challenges associated with aging process. One of the challenges involves the deterioration in older people's cognitive functioning with reduced working memory capacity and processing speed [1]. Mikels, Shuster, and Thai [2] further point out that cognitive deteriorations may result in unintended consequences in emotion such as chronological depression, lack of interest which can significantly affect the quality of older people's life. Previous research has established that the decline of older people's abilities in information processing may be compensated through multiple sensory inputs in multi-modal learning since visual processing is found to be a common factor shared among cognition, speed, and sensory functions for older adults [3-5]. Differing from the visual compensatory research, studies in $g$ intelligences find that crystalized intelligence - one of the $g$ intelligences - is correlated with older people's cognitive performance in problem solving $[1,6,7]$. Crystallized intelligence refers to an individual's cumulative knowledge often known as schema and prior knowledge [8] and is believed to be crucial in meaningful cognitive learning [9]. Despite our knowledge in older people's visual processing and the role of crystalized intelligence in learning, research in this area is still undertheorized. In addition, many of the empirical findings related to older people's multi-modal learning and $\mathrm{g}$ intelligences are based on anecdotal evidences like solving crossword puzzles [7] which is tangential to formal, curriculum-based learning. It is arguable whether the above findings can be generalized to a formal learning setting when developing training for older people. The goals of the current paper are to understand (1) the relationship between multi-modal learning and $\mathrm{Gc}$ activation and (2) the strategies effective for delivering training for older adults.

\section{Theoretical Background}

It has been widely recognized that human's abilities to process information is largely constrained by the working memory capacity $[10,11]$. This situation gets aggravated with the aging process where older people constantly experience short-term memory loss and failing to perform simultaneously several tasks at one time. According to Baddeley [10], input information such as auditory and visual information is processed through a temporary storage before it is encoded into the long-term memory. This temporary storage known as working memory, is characterized by a central executive function which has two sub-systems: phonological loop and visuo-spatial sketchpad (VSS). The phonological loop stores phonological information like sound and prevents its decay by silently articulating its contents, thereby refreshing the information in a rehearsal loop. The VSS is believed to process and manipulate visuo-spatial images [12]. For example, the ability to mentally manipulate 3D images by rotating them in the mind is largely determined by the VSS function. The central executive function carries out the cognitive activities such as reasoning and problem-solving by coordinating the information from phonological loop and visuo-spatial skethpad. Baddeley's working memory theory is credited for its contribution to the advancement of several theoretical development in multimedia and multi-modal learning [13, 14]. Studies show that the working memory is very limited in both duration and capacity. Van Merrienboer and Sweller [15] observed that the working memory stores about seven elements but normally operates on only two or three elements. When the working memory becomes overloaded with information, learning can be adversely affected [16, 17]. 


\subsection{Efforts to Improve Working Memory Performance}

One of the approaches in improving working memory performance is to introduce multimedia with multiple sensory input modes (e.g., visuals, audio) to learning $[9,18]$. Mayer's cognitive theory of multimedia learning (CTML) posits that information that is processed through multiple sensory modes (multi-modal learning) helps alleviate the amount of cognitive load in learners' working memory, increase cognitive resources and contribute to the formation of mental representation [19-21]. Imagine reading a medical text in vascular system and anatomy. If the information is presented in text only, the reader may have trouble comprehending how the vascular system works. In other words, information that is processed through mono-mode (i.e., text) can easily overload the VSS system in the working memory. However, instead of presenting the information with only text, the information is delivered through text and pictures (multi-modal) which means the same amount of information is processed separately through text and image which alleviate the pressure on working memory and make cognitive resources available during learning. Mayer and colleagues $[18,22,23]$ conduct several studies to test the modality effect in learning. They find that learning with multiple modes (image, sound) is better than learning with a single mode (text).

\subsection{Redundancy Hypotheses}

With multi-modal learning showing a superior effect over mono-modal learning, the question then becomes, would adding more presentation modes lead to better performance in learning? Mayer and colleagues [13, 24] study the effects of multi-modal learning (e.g., text, animation, and audio) on learners' performance as measured by information retention and transfer. In one condition the learners are presented with text, animation and narration to study a subject in science whereas in another condition only animation and narration are provided. The researchers observe that learners who study with animation and narration perform better than these who study with text, animation and narration. They conclude that the redundant text may interfere with learners' information processing as they have to switch their attention between the text and animation. Mayer [13] concludes that visuals like text and animation, when presented simultaneously with narration, can cause an interference in information processing and result in an overload in working memory - a phenomenon Mayer describes as redundancy effect in multimedia learning [18].

The redundancy hypothesis has been widely tested in education and corporate settings. For example, Jamet and Le Bohec [25] examine three groups of learners with one group having no written text, the second group having written sentences redundant with the spoken information showing progressively on the screen, and the third group having these written sentences presented together. Their results show whatever the type of text presentation (sequential or static), the duplication of information in the written mode results in a substantial impairment in subsequent retention and transfer tests as well as in a task in which the memorization of diagrams is evaluated. The study conducted by McNeill, Doolittle, and Hicks [26] corroborates Jamet and Le Bohec's findings. McNeill et al. examine the effects of narration with identical onscreen text in science learning. The results show an impairment for learning with narration and identical onscreen text. Contrary to the previous findings, Samur [27] finds adding on-screen text to a 
presentation with animation and narration helps students learn new vocabulary of a previously unfamiliar foreign language. Samur's study involves learners who study a foreign language with little or no background knowledge. It is possible that the redundant information from text and narration may compensate their lacking in schemas. Samur's study suggests that when one of the sensory process (e.g., auditory) becomes less functional due to reasons like lacking prior knowledge or reduced processing functioning, adding another sensory process (e.g., visual) may compensate the diminishing function of the primary sensory process. Samur's finding is important in that it sheds light on the relationship between modes of presentation and sensory functioning.

\subsection{Compensatory Hypothesis}

Initial evidence has shown that the diminishing function of primary sensory process may be compensated by adding a secondary sensory process in learning [27]. This is particularly true for older people. For example, Anstey et al [3] study the older people's sensory functioning in relation to their cognitive processes. It is found that the memory which is an important indicator in older people's cognitive processing is heavily loaded onto sensory functions. This means older people's abilities to process information can be significantly affected by their sensory functioning. In a cross-sectional-age study Bates and Lindenberger [4] compare the older people with young population (age range: 25-103 years) in terms of sensory functioning and intellectual abilities. Of five different intellectual abilities (e.g., memory, reasoning, processing speed), two of major sensory functions (i.e., vision and hearing) are found to be strong predictors for intellectual abilities with $31 \%$ of the total variances for the older sample (age range: $70-103$ years) compared to $11 \%$ of the total variances for the younger sample (age range: $25-69$ years). What this means is that when processing information older people tend to rely on more sensory inputs in order to make sense of the new content. Researchers thus hypothesize that given the association between older persons' cognition and sensory functions, the diminishing cognitive abilities in older people, like working memory deficits, can be compensated by extra sensory support, which is known as the Compensatory Hypothesis [3, 4, 28]. The significance of compensatory hypothesis lies in (1) explaining the role of redundant visuals from the perspective of aging process, and (2) modifying redundancy hypothesis by showing that redundant visuals may not necessarily be detrimental to older learners due to a compensatory effect.

\section{The Role of $G$ Factor in Older People's Learning}

It is widely recognized that general intelligences, known as $g$ factor, can influence individual performance in learning [29]. There are eight sub-types of $g$ intelligences which include fluid intelligence (Gf), crystallized intelligence (Gc), general memory and learning (Gy), broad visual perception (Gv), broad auditory perception (Gu), broad retrieval ability (Gr), broad cognitive speediness (Gs), and processing speed (Gt) [8]. Research has demonstrated that Gc and Gf are the two intelligences that are critically related to older people's learning $[1,7,30]$. Salthouse and colleagues conduct a study on older people's crossword puzzle problem solving and find Gc is significantly correlated with their problem solving abilities. In the following section the discussion will be focused on the roles of Gc and Gf in older people's learning. 


\subsection{Gc and Gf Intelligences}

Crystallized and fluid intelligences, also known as Gc and Gf intelligences respectively, are closely related to an individual's ability in task performance. Gc reflects one's lifetime of intellectual achievement, as demonstrated largely through one's prior knowledge and schema whereas Gf relates to one's creative thinking and reasoning ability [31]. Studies show that Gf starts to decline in the mid-20s, with a significant downturn beginning at age 50, whereas Gc increases over time, reaching a plateau at the age of 50 [32]. Salthouse [7] conducts several studies on older people's abilities in problem solving (e.g., crossword puzzles). He finds that the older people's abilities in problem solving decline when they solve novel problems since, he argues, solving novel problems requires creative and innovative thinking. This finding is consistent with the literature that people's fluid intelligence (Gf) declines as they get older. However, when knowledge becomes relevant to the task, as in crossword puzzles, the older people with frequent crossword puzzlesolving experience actually show better performance. This indicates that for older people, much of the effective power of the learning derives from an accumulated fund of information (Gc), and that any loss of creative and innovative abilities (Gf) can be compensated for by higher levels of experience and knowledge. This finding is significant in that it raises the awareness of (a) the important role of $\mathrm{Gc}$ in older adults' information processing and (b) its utility in older adults' training.

\subsection{Cognitive Prompts for Gc Activation}

If Gc, as Salthouse [7] points out, is an important factor in older people's learning, identifying the strategies that activate Gc has some significance with respect to the design and development of effective training for older people. Cognitive prompts have recently drawn attention in aging related research $[33,34]$. Cognitive prompts are learning questions placed throughout an educational presentation - be they in person or through multimedia - that aim at activating prior knowledge or focusing on the learners' attention during learning. Cognitive prompts can support both cognitive processes - including memory retrieval - and metacognitive development in learning [35]. Studies show that cognitive prompts can improve knowledge transfer significantly by directing learners' attention to structural features that are related to previously learned information [36].

Preliminary evidence suggests that cognitive prompts help activate learners' prior knowledge/schemas, thus improving learners' abilities to comprehend the content and apply the knowledge to new learning [35, 37]. Wolfson et al [34] suggest that technology-based training may benefit from incorporating cognitive prompts to direct older adults' attention to key information to avoid unnecessary processing in learning. This view is further supported by Colombo and Antonietti's study [33], which demonstrates that cognitive prompts help the older learners relate previously learned information to the present task through prior knowledge/schema activation. Given the relationship between cognitive prompts and information processing in older adults' learning, it can be reasonably assumed that the knowledge activation in schemas through cognitive prompts will enable the older learners to engage in more meaningful learning. In addition, more cognitive resources will be available since the information retrieved from the schema is packaged in units, which consequently reduces the processing load in working memory 
[38]. Regardless, studies that focus on formal training for older people in regard to Gc activation in multi-modal learning is lacking. Therefore, more research is warranted.

\section{A Framework for Multi-Modal Learning and Gc Activation}

The above review on research in multi-modal learning and $\mathrm{Gc}$ activation reveals a common underlying theoretical theme: the older people's learning is largely affected by the available cognitive resources in working memory. To ensure that the older people have sufficient cognitive resources during learning, researchers have studied instructional strategies aiming at optimizing older people's learning performance. Based on Mayer's [13] studies in modality and redundancy, researchers further explore the functional role of redundant multimedia by taking into perspective aging process. The compensatory hypothesis explains how older people may benefit from multimodal learning with redundant visuals (redundancy effect) since they need extra sensory inputs to compensate the declining visual and hearing abilities. In other words, the redundancy effect in multimedia turns out to be beneficial for older people due to a compensatory effect. Meanwhile, research reveals that $\mathrm{Gc}$ intelligence which is one of $\mathrm{g}$ intelligences plays a key role in older people's learning. By activating Gc intelligence, older people will make meaningful connection between new information and schemas which in turn makes cognitive resources available in working memory due to the load reduction in information processing. Although multi-modal learning and Gc activation hold promises in the research for older adults' learning, there is a gap between them. Connecting two areas of studies will improve our understanding of the roles of multi-modal learning and Gc activation. Figure 1 presents a framework which describes the relationship between multi-modal learning and Gc activation. The proposed framework, called multi-modal - Gc activation model, explicates how older people's mental representation can be supported by text, images and sound (compensatory effect) and be strengthened by the activation of Gc.

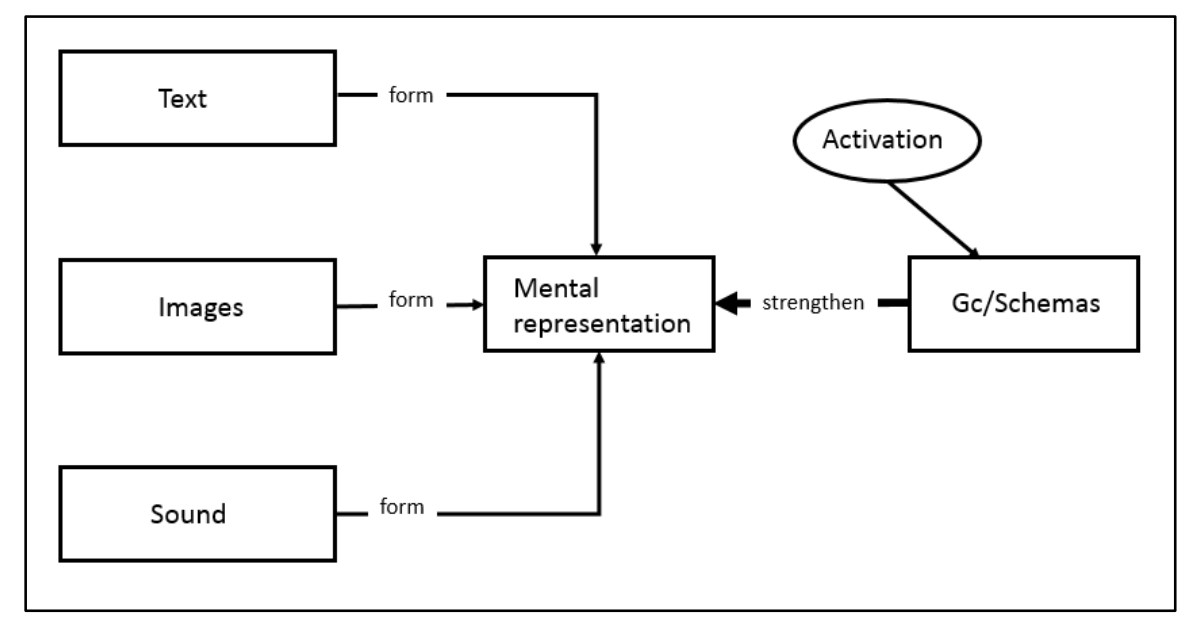

Figure 1 Multi-modal - Gc activation model for older adults' learning.

\section{Evidences in Support of Multi-Modal - Gc Activation Model}

In the next section, findings from initial empirical studies will be discussed in support of the proposed multi-model - Gc activation model. 


\subsection{Evidence of Compensatory Effect in Older People's Caregiving Training}

Redundancy hypothesis and compensatory hypothesis are two contradictory theoretical frameworks, both of which have significant implications in the design of learning materials for older people. Based on redundancy hypothesis, it is suggested that multimedia should avoid placing simultaneously text and animation with narration. In contrast, compensatory hypothesis suggests that simultaneous presentation of text, animation, and narration can benefit older people since their diminishing abilities in one cognitive function can be compensated by additional information processed through another sensory input. To test both hypotheses, Zheng et al [39] conduct a cross-sectional-age study $(N=82)$ with age ranging from 18 to 85 years old. Participants are randomly assigned to redundancy and non-redundancy learning conditions in caregiving training. In the redundancy condition, participants are presented with video, narration and identical on-screen text. In the non-redundancy condition, participants are presented with only video and narration without on-screen text. For the younger population, the results confirm the redundancy hypothesis that participants in non-redundancy condition outperform these in redundancy condition as measured by recall and knowledge transfer. However, for the older population a reversed effect is observed with participants performing better in the redundancy condition than in the non-redundancy condition which confirms the compensatory hypothesis that "older persons may need extra sensory support in information processing at the cost of redundancy due to their declines in working memory and processing speed" [39].

The challenge with compensatory hypothesis is that it is difficult to account for all the cognitive processes based on multiple sensory inputs [4]. For example, mental representation can be influenced by an individual's schema. Given the research on the relationship between $\mathrm{Gc}$ and older people's abilities in problem solving [1, 7, 30, 40], Smith et al [41] conduct a study to further examine the effect of Gc activation in multi-modal learning.

\subsection{Evidence of Gc Activation in Older People's Mental Representation}

Evidence from empirical studies demonstrate the benefits of multiple visual stimuli in compensating the declining cognitive abilities of older people in learning [39]. To understand if the activation of Gc intelligence would facilitate older people's mental representation in multi-modal learning, Smith et al [41] examine the relationship between cognitive prompts, Gc factor, and older people's abilities to form mental representation in caregiving training, specifically how the activation of Gc would facilitate older learners' mental representation and their abilities to process multiple modes of information in caregiving training. The study involves sixty older people (Mage=69.47). Two conditions are created (cognitive prompts vs. no cognitive prompts) to test the presence of cognitive prompts in Gc activation in multi-modal learning. The findings reveal a significant correlation between cognitive prompts and Gc intelligence. The path analysis further shows that $\mathrm{Gc}$ is a significant mediator that mediates between cognitive prompts and learning performance as measured by recall and transfer tests. It is also found that by activating their schema, older people are able to make meaningful connections between their prior knowledge and new materials regardless of their declining working memory capacity and processing speed. Smith et al.'s study provides initial evidence with respect to the functional role of Gc in older people's mental representation in complex learning. 


\section{Discussions and Conclusion}

The goal of this paper is to understand the functional roles of multi-modal learning and Gc activation in older people's learning. Two different theoretical frameworks are discussed with a focus on the constraints of working memory during information processing in learning. The multimodal learning framework is based on the assumption that multiple sensory inputs may free the processing pressure and thus support older people's learning. However, implementing multimodal learning framework can be complicated as multiple sensory inputs could create a redundancy effect. From the compensatory hypothesis we learn that redundant sensory input can benefit older people's learning as their diminishing cognitive processing abilities can be compensated by extra visuals. The Gc activation framework accentuates the importance of schema in older people's learning. Strategies like cognitive prompts are commonly used to facilitate the Gc activation. What is missing in current research is the connection between multimodal learning and Gc activation. Thus, a framework called multi-model - Gc activation model is proposed aiming to build the connection between multi-modal learning and Gc activation. The initial evidence has shown that older people's ability to form mental representation can be facilitated by multiple sensory inputs confirming the compensatory hypothesis that adding redundant visuals compensates the older people's diminishing cognitive processing abilities in learning. A related follow-up study further provides evidence that activating older people's Gc supports their mental representation and abilities in processing multi-mode information evincing a link between Gc and multi-modal learning.

The findings of above studies have significant implications in the design and development of training for older people. First, they confirm the benefits of multi-modal learning for older people, in this case, the caregiving training. Second, the studies show that the redundant visuals are relevant and beneficial to older people's learning, supporting compensatory hypothesis. Third, it identifies the connection between multi-modal learning and Gc activation in terms of the formation of mental representation in older people's learning. At the practical level, the findings of the studies offer some insights on the curricular design in older people training.

\subsection{Recommendations}

It is recommended that the curriculum for older people training should consider (1) using multiple sensory modes to facilitate older people's information process and (2) directing attention to the role of redundant visuals to support older people' learning based on compensatory hypothesis, and (3) employing cognitive prompts to activate older people's schemas so they can engage in meaningful learning.

1. Using multiple sensory modes to facilitate older people's information process. It is recommended that when designing training for older people, the trainer should employ various media (e.g., visual, audio, and interactive devices) to support older people's learning who often experience some level of impairments in cognitive functioning and sensory motor skills. For example, learning personal financial management can be challenging for older people. The training may incorporate multiple visuals (e.g., text and diagram) to facilitate the understanding of the concepts like sources of incoming (e.g., personal savings, investment, 401K, and Social Security funds) and spending (e.g., food, 
vacation, recreation, medical expenses). The above process of concept learning can be further strengthened by including interactive activities involving multiple sensory inputs which can occur in both computer-based platform and face-to-face environments using strategies like role-playing, simulation, and modeling to help older people understand the relationships among the concepts.

2. Considering the role of redundant visuals to support older people' learning based on compensatory hypothesis. The literature has shown that redundant visuals can be beneficial to older people's learning despite the fact that redundancy may cause a cognitive overload in young people's information process. Because of their declines in both cognitive and sensory performances, the training for older people should be designed in such a way that they are able to process information through multiple sensory channels with multiple stimuli. For example, when learning how to use social media which research has found a significant increase in use among older people [5], the training may include narration, video, and on-screen captions to show the steps of using social media (e.g., using Facebook to communicate with their grandchildren). Other strategies like group activity, instructional guides can be effective supplements to the above training where older people can experience the dynamics of social media via group interaction or control individual pace of learning by following the instructional guides.

3. Employing cognitive prompts to activate older people's schemas. The research has shown that the power of older people's learning derives much from their funds of knowledge. Cognitive prompts can be an effective way to activate and utilize older people's funds of knowledge in learning. For example, Zheng et al [39] found cognitive prompts can promote both surface and deep level understanding in older people's caregiving training. Strategies like embedding short narratives or questions in the training material can provide additional context to connect to learners' schemas. The following is an example of cognitive prompts for dementia caregiving training. The cognitive prompts are embedded within the training video aiming to help the learners make the connection between the caregiving content and their schemas.

STAGES OF DEMENTIA: (Prompts approx. $16 \mathrm{sec})$

Doctor says, "Socializing is good for you"

PROMPT: Imagine ways that socializing can improve quality of life for someone with dementia.

Doctor says, "Stick with hobbies you've enjoyed all your life; anything that stimulates the brain helps"

PROMPT: Imagine activities that can improve quality of life for someone with dementia

Doctor says, "Hoarding, repetitive movements, paranoia, emotional - even physical outbursts..."

PROMPT: Think of other unusual behaviors that dementia patients may show at a moderate or severe stage.

Cognitive prompts can also be implemented via supporting groups where learners help each other by asking questions or providing background information relating to the topic and background information. 
To further substantiate the above recommendations, a table that delineates the connections between recommendations and their underlying theoretical constructs is provided with empirical evidences to support the recommendations and the underlying theoretical constructs (Table 1).

In sum, both multi-modal and Gc activation approaches have demonstrated the effectiveness in older people's learning by overcoming working memory constraints with aging. It is suggested that more research is needed to understand the relationship between multi-modal learning and Gc activation. More research should be conducted to understand the relationship between redundant visual processing and Gc activation, that is, how many sensory inputs can we provide at once time in older people's learning? How to optimally utilize the cognitive resources released from Gc activation to mental representation? Finally, future efforts should be directed toward the generalization of the findings to a broader population in terms of ethnicity, gender, individual difference, and social-economic status.

Table 1 Empirical evidences supporting recommendations for multi-modal learning and Gc activation for older adults.

\begin{tabular}{|c|c|c|}
\hline Recommendations & Theoretical Constructs & Studies \\
\hline $\begin{array}{l}\text { Multiple sensory } \\
\text { modes to facilitate } \\
\text { older people's } \\
\text { information } \\
\text { process }\end{array}$ & $\begin{array}{l}\text { - } \text { Multi-modal processing } \\
\text { modfectiveness of Multi- } \\
\text { people learning }\end{array}$ & $\begin{array}{l}\text { Simpson, T., Camfield, D., Pipingas, } \\
\text { A., Macpherson, H., \& Stough, C. Improved } \\
\text { processing speed: Online computer-based } \\
\text { cognitive training in older adults. Educational } \\
\text { Gerontology. 2012: 38(7), 445-458. } \\
\text { Lindenberger, U., \& Baltes, P.B. Sensory } \\
\text { functioning and intelligence in old age: A } \\
\text { strong connection. Psychology and Aging. } \\
\text { 1994: 9, 339-355. }\end{array}$ \\
\hline $\begin{array}{l}\text { Redundant visuals } \\
\text { relevant to older } \\
\text { people's learning } \\
\text { due to a } \\
\text { compensatory } \\
\text { effect }\end{array}$ & $\begin{array}{ll}\text { - } & \text { Redundancy effect } \\
\text { - } & \text { Compensatory effect }\end{array}$ & $\begin{array}{l}\text { Zheng, R., Smith, D., Luptak, M., Hill, R., Hill, J., } \\
\text { \& Rupper, R. Does visual redundancy inhibit } \\
\text { older persons' information processing in } \\
\text { learning? Educational Gerontology. 2016: 42, } \\
635-645 .\end{array}$ \\
\hline $\begin{array}{l}\text { Cognitive prompts } \\
\text { to activate older } \\
\text { people's schemas } \\
\text { for meaningful } \\
\text { learning }\end{array}$ & $\begin{array}{l}\text { - Schema/crystalized } \\
\text { intelligences (Gc) } \\
\text { - Activation of schema/ } \\
\text { crystalized intelligence } \\
\text { (Gc) in multimodal } \\
\text { learning }\end{array}$ & $\begin{array}{l}\text { Smith, M. A., Blunt, J. R., Whiffen, J. W., \& } \\
\text { Karpicke, J. D. Does providing prompts during } \\
\text { retrieval practice improve learning? Applied } \\
\text { Cognitive Psychology. 2016: 30(4), 544-553. } \\
\text { Smith, D., Zheng, R., Metz, A. J., Morrow, S., } \\
\text { Pompa, J., Hill, J., \& Rupper, R. Role of } \\
\text { cognitive prompts in video caregiving training } \\
\text { for older adults: Optimizing deep and surface } \\
\text { learning. Educational Gerontology. } 2019 \text {. } \\
\text { Umanath, S., \& Marsh, E. J. Understanding } \\
\text { how prior knowledge influences memory in } \\
\text { older adults. Perspectives on Psychological } \\
\text { Science. 2014: 9(4), 408-426. }\end{array}$ \\
\hline
\end{tabular}




\section{Author Contributions}

The author wrote and revised the manuscript.

\section{Competing Interests}

The author has declared that no competing interests exist.

\section{References}

1. Salthouse TA. Consequences of age-related cognitive declines. Annu Rev Psychol. 2012; 63: 201-226.

2. Mikels JA, Shuster MM, Thai ST. Aging, emotion, and decision making. In H. M. Thomas, Strough, J., Löckenhoff, C. E. (Eds); Aging and decision making: Empirical and applied perspectives (pp. 169-188). Cambridge, MA: Elsevier Academic Press. 2015.

3. Anstey KJ, Luszcz MA, Sanchez LA. Reevaluation of the common factor theory of shared variance among age, sensory function, and cognitive function in older adults. J Gerontol B: Psychol Sci Soc Sci. 2001; 56: 3-11.

4. Bates PB, Lindenberger $U$. Emergence of a powerful connection between sensory and cognitive functions across the adult life span: A new window to the study of cognitive aging? Psychol Aging. 1997; 12: 12-21.

5. Zheng R, Spears J, Luptak M, Wilby F. Understanding older adults' perceptions of Internet use: An exploratory factor analysis. Educ Gerontol. 2015; 41: 504-518.

6. Lindenberger $U$, Baltes PB. Sensory functioning and intelligence in old age: $A$ strong connection. Psychol Aging. 1994; 9: 339-355.

7. Salthouse TA. Major issues in cognitive aging. New York: Oxford University Press. 2010.

8. Carroll JB. Psychometrics, intelligence, and public perception. Intelligence. 1997; 24: 25-52.

9. Zheng R, Yang W, Garcia D, McCadden BP. Effects of multimedia on schema induced analogical reasoning in science learning. J Comput Assist Learn. 2008; 24: 474-482.

10. Baddeley AD. Working memory. Science. 1992; 255: 556-559.

11. Miller GA. The magical number seven, plus or minus two: Some limits on our capacity for processing information. Psychol Rev. 1956; 63: 81-97.

12. Baddeley AD, Thompson N, Buchanan M. Word length and the structure of memory. J Verbal Learn Verbal Behav. 1975; I: 575-589.

13. Mayer RE. Multimedia learning. Cambridge, United Kingdom: Cambridge Press. 2001. (pp. 1201).

14. Zheng R. (Ed.). Cognitive effectives of multimedia learning. Hershey, PA: Information Science Reference/IGI Global Publishing. 2008 (1-xx).

15. Van Merrienboer JJG, Sweller J. Cognitive load theory and complex learning: Recent developments and future directions. Educ Psychol Rev. 2005; 17: 147-177.

16. Marcus N, Cooper M, Sweller J. Understanding instructions. J Educ Psychol. 1996; 88: 49-63.

17. Sweller J, Chandler P. Why some material is difficult to learn. Cognition and Instruction. 1994; 12: 185-233.

18. Mayer RE, Moreno R. Nine ways to reduce cognitive load in multimedia learning. J Educ Psychol. 2003; 38: 43-52. 
19. Paivio A. Mental representations: A dual coding approach. Oxford, United Kingdom: Oxford University Press. 1986.

20. Zheng, R., \& Cook, A. Solving complex problems: A convergent approach to cognitive load measurement. British Journal of Educational Technology, 2012: 43(2), 233-246.

21. Zheng $R$, McAlack $M$, Wilmes $B$, Kohler-Evans $P$, Williamson J. Effects of multimedia on cognitive load, self-efficacy, and multiple rule-based problem solving. Brit J Educ Technol. 2009; 40: 790-803.

22. Mayer RE, Anderson RB. Animations need narrations: An experimental test of a dual coding hypothesis. J Educ Psychol. 1991; 83: 484-490.

23. Mayer RE, Johnson $\mathrm{Cl}$. Revising the redundancy principle in multimedia learning. J Educ Psychol. 2008; 100: 380-386.

24. Moreno R, Mayer RE. Cognitive principles of multimedia learning: The role of modality and contiguity. J Educ Psychol. 1999; 91: 358-368.

25. Jamet $E$, Le Bohec $O$. The effect of redundant text in multimedia instruction. Contemp Educ Psychol. 2007; 32: 588-598.

26. McNeill AL, Doolittle PE, Hicks D. The effects of training, modality, and redundancy on the development of a historical inquiry strategy in a multimedia learning environment. J Interact Online Learn. 2009; 8: 255-269.

27. Samur Y. Redundancy effect on retention of vocabulary words using multimedia presentation. Brit J Educ Technol. 2012; 43: 166-170.

28. Christensen H, Mackinnon AJ, Korten A, Jorm AF. The common cause hypothesis of cognitive aging: Evidence for not only a common factor but also specific associations of age with vision and grip strength in a cross-sectional analysis. Psychol Aging. 2001; 16: 588-599.

29. Blanch A. Evaluating fluid and crystallized abilities in the performance of an educational process. Instructional Sci. 2015; 43: 427-442.

30. Hambrick DZ, Salthouse TA, Meinz EJ. Predictors of crossword puzzle proficiency and moderators of age-cognition relations. J Exp Psychol: General. 1999; 128: 131-164.

31. Benson N, Hulac DM, Kranzler JH. Independent examination of the Wechsler adult intelligence scale - fourth edition (WAIS-IV): What does the WAIS-IV measure? Psychol Assess. 2010; 22: 121-130.

32. Horn JL, Donaldson G, Engstrom R. Apprehension, memory, and fluid intelligence decline in adulthood. Res Ageing. 1981; 3: 33-84.

33. Colombo B, Antonietti $A$. The role of metacognitive strategies in learning music: $A$ multiple case study. Brit J Music Educ. 2017; 34: 95-113.

34. Wolfson NE, Cavanagh TM, Kraiger K. Older adults and technology-based instruction: Optimizing learning outcomes and transfer. Acad Manage Learn Educ. 2014; 13: 26-44.

35. Berthold K, Nuckles M, Renkl A. Do learning protocols support learning strategies and outcomes? The role of cognitive and metacognitive prompts. Learning and Instruction. 2007; 17: 564-577.

36. Monks T, Robinson S, Kotiadis K. Can involving clients in simulation studies help them solve their future problems? A transfer of learning experiment. Eur J Oper Res. 2016; 249: 919-930.

37. Hsu YS, Wang CY, Zhang WX. Supporting technology-enhanced inquiry through metacognitive and cognitive prompts: Sequential analysis of metacognitive actions in response to mixed prompts. Comput Human Behav. 2017; 72: 701-712. 
38. Logan GD. Automaticity and memory. In W. E. Hockley and S. Lewandowsky (Eds), Relating theory and data: Essays on human memory in honor of Bennet B. Murdock. Lawrence Erlbaum Associates. 1991; 347-366.

39. Zheng R, Smith D, Luptak M, Hill R, Hill J, Rupper R. Does visual redundancy inhibit older persons' information processing in learning? Educ Gerontol. 2016; 42: 635-645.

40. Badham SP, Hay M, Foxon N, Kaur K, Maylor EA. When does prior knowledge disproportionately benefit older adults' memory? Ageing Neuropsychol Cogn. 2016; 23: 338365.

41. Smith D, Zheng R, Metz AJ, Morrow S, Pompa J, Hill J, et al. Role of cognitive prompts in video caregiving training for older adults: Optimizing deep and surface learning. Educ Gerontol. 2019.

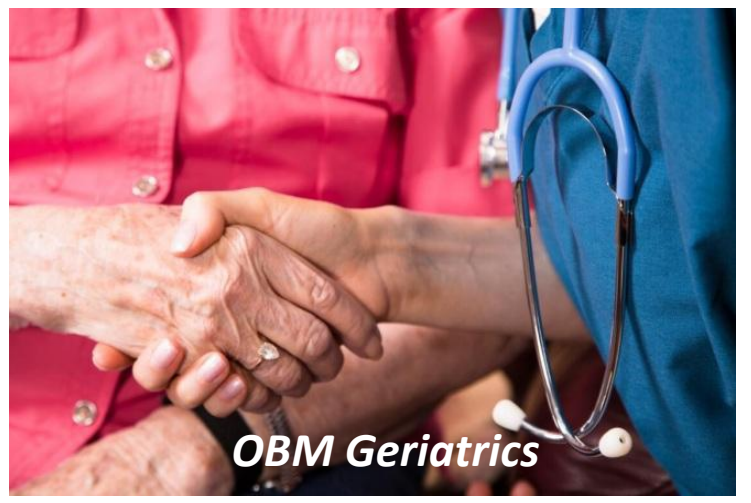

Enjoy OBM Geriatrics by:

1. Submitting a manuscript

2. Joining in volunteer reviewer bank

3. Joining Editorial Board

4. Guest editing a special issue

For more details, please visit:

http://www.lidsen.com/journals/geriatrics 